\title{
ORIGINAL ARTICLE \\ The effect of neighborhood size on effective population size in theory and in practice
}

\author{
L Nunney
}

The distinction between the effective size of a population $\left(N_{\mathrm{e}}\right)$ and the effective size of its neighborhoods $\left(N_{\mathrm{n}}\right)$ has sometimes become blurred. $N_{\mathrm{e}}$ reflects the effect of random sampling on the genetic composition of a population of size $N$, whereas $N_{\mathrm{n}}$ is a measure of within-population spatial genetic structure and depends strongly on the dispersal characteristics of a species. Although $N_{\mathrm{n}}$ is independent of $N_{\mathrm{e}}$, the reverse is not true. Using simulations of a population of annual plants, it was found that the effect of $N_{\mathrm{n}}$ on $N_{\mathrm{e}}$ was well approximated by $N_{\mathrm{e}}=N /\left(1-F_{\mathrm{IS}}\right)$, where $F_{\mathrm{IS}}$ (determined by $N_{\mathrm{n}}$ ) was evaluated population wide. $N_{n}$ only had a notable influence of increasing $N_{\mathrm{e}}$ as it became smaller $(\leqslant 16)$. In contrast, the effect of $N_{\mathrm{n}}$ on genetic estimates of $N_{\mathrm{e}}$ was substantial. Using the temporal method (a standard two-sample approach) based on 1000 single-nucleotide polymorphisms (SNPs), and varying sampling method, sample size (2-25\% of $N$ ) and interval between samples ( $T=1-32$ generations), estimates of $N_{\mathrm{e}}$ ranged from infinity to $<0.1 \%$ of the true value (defined as $N_{\mathrm{e}}$ based on $100 \%$ sampling). Estimates were never accurate unless $N_{\mathrm{n}}$ and $T$ were large. Three sampling techniques were tested: same-site resampling, different-site resampling and random sampling. Random sampling was the least biased method. Extremely low estimates often resulted when different-site resampling was used, especially when the population was large and the sample fraction was small, raising the possibility that this estimation bias could be a factor determining some very low $N_{\mathrm{e}} / N$ that have been published. Heredity (2016) 117, 224-232; doi:10.1038/hdy.2016.76; published online 24 August 2016

\section{INTRODUCTION}

Two measures that help us predict the influence of genetic drift on a population are the (effective) neighborhood number $\left(N_{\mathrm{n}}\right)$ and effective population size $\left(N_{\mathrm{e}}\right)$. However, the distinction between them is often misunderstood and here my aim is to clarify their relationship, emphasizing that these two measures are conceptually very different. $N_{\mathrm{e}}$ is the effective number of individuals within the whole population, defined as the size of an ideal population affected by random genetic sampling at the same rate as the population being studied (Wright, 1931,1938), whereas $N_{\mathrm{n}}$ is the effective number of individuals within an area of a population (the neighborhood) defined such that the parents of a focal individual can be considered to be genetically representative of the neighborhood's occupants (Wright, 1946). To avoid confusion, the notation $N_{\mathrm{n}}$ is used for the neighborhood number instead of $N_{\mathrm{b}}$ (Slatkin and Barton, 1989) as $N_{\mathrm{b}}$ has been used for the effective number of breeders in a given year in species with overlapping generations (Waples, 2005), and, more generally, for the effective number of parents producing the sample used in the estimation of $N_{\mathrm{e}}$ when there is temporal or spatial population structure (Neel et al., 2013).

Both $N_{\mathrm{e}}$ and $N_{\mathrm{n}}$ increase with the density of individuals within a population, and some of the species-specific factors that influence $N_{\mathrm{e}}$ by altering the variance in reproductive success (Wright, 1938) such as longevity and mating system (Nunney, 1993) can also influence $N_{n}$; however, the two measures differ in scale and, as a result, they differ in the nature of their influence on the genetic composition of a population. A major factor determining $N_{\mathrm{e}}$ is the size of the population $(N)$, and that is largely dependent upon the area of available habitat. In contrast, $N_{\mathrm{n}}$ is independent of $N$ as it is defined at a local scale by the dispersal biology of the species. Any population exhibiting spatial structure must encompass an area greater than a single neighborhood, as when this is not the case the population is panmictic.

$N_{\mathrm{e}}$ is expected to vary substantially across populations because of its strong dependence upon the area of suitable habitat availability, an area that can vary enormously from population to population, whereas $N_{\mathrm{n}}$ is expected to vary little among populations of the same species given the relative constancy of species-specific dispersal patterns. Moreover, as $N_{\mathrm{e}}$ is affected by the spatial structure of a population (Whitlock and Barton, 1997; Nunney, 1999), $N_{\mathrm{n}}$ can directly affect $N_{\mathrm{e}}$, whereas the reverse is not true.

The rate at which neutral genetic variation is lost from a population because of genetic drift is determined by the effective population size $\left(N_{\mathrm{e}}\right)$. Although this measure is primarily dependent upon $N$, it is modified by a variety of factors (see Hare et al., 2011). One such factor is nonrandom mating. Wright (1943) showed how two different sources of nonrandom mating affect $N_{\mathrm{e}}$ under otherwise ideal conditions. First, given local inbreeding (such as selfing or brother/ sister mating) but no spatial structure, then $N_{\mathrm{e}}=\mathrm{N} /\left(1+F_{\mathrm{IS}}\right)$; and second, given a spatial structure of semi-isolated island sub-populations (and random mating within each), $N_{e}=N /\left(1-F_{\mathrm{ST}}\right)$. In these formulae, $F_{\mathrm{IS}}$ and $F_{\mathrm{ST}}$ are the hierarchical inbreeding coefficients (Wright, 1951). These two results can be combined into 
a single relationship (Nunney, 1999):

$$
N_{\mathrm{e}}=N /\left[\left(1+F_{\mathrm{IS}}\right)\left(1-F_{\mathrm{ST}}\right)\right]
$$

that illustrates how $F_{\mathrm{IS}}$, derived from local inbreeding, and $F_{\mathrm{ST}}$, derived from the isolation of the island sub-populations, influence $N_{\mathrm{e}}$ in opposite ways.

This observation that $F_{I S}$ generated by a regular system of inbreeding acts to reduce $N_{\mathrm{e}}$ extends to nonideal populations (see Caballero and Hill, 1992; Wang, 1996; Yonezawa, 1997; Nunney, 1999); however, the effect of the larger scale inbreeding due to the island subdivisions $\left(F_{\mathrm{ST}}\right)$ depends on the model used. The influence of $F_{\mathrm{ST}}$ in the island model in increasing $N_{\mathrm{e}}$ (Equation (1)) is dependent upon the assumption that island productivity is locally regulated, such that all islands contribute equally to the dispersal pool (Whitlock and Barton, 1997). If, on the other hand, a demic model is used, where population regulation allows productivity differences among the demes (or islands) to be reflected in the pool of migrants, then these productivity differences generate interdemic genetic drift. The result is that the influence of $F_{\mathrm{IS}}$ and $F_{\mathrm{ST}}$ become identical (Nunney, 1999):

$$
N_{\mathrm{e}}=N /\left[\left(1+F_{\mathrm{IS}}\right)\left(1+F_{\mathrm{ST}}\right)-2 F_{\mathrm{IS}} F_{\mathrm{ST}}\right]
$$

This difference between the effective size of a metapopulation with equal dispersal per island (island model, Equation (1)) versus one with varying dispersal from each island (demic model, Equation (2)) is important in defining conservation strategies (see, for example, Gilpin, 1991; Hedrick and Gilpin, 1996; Whitlock and Barton, 1997; Nunney, 2000).

The Equations (1) and (2) provide the basis for understanding how $N_{e}$ is affected when a deviation from Hardy-Weinberg ratios occurs either as a result of a regular system of close inbreeding combined with uniform dispersal $\left(=F_{I S}\right)$, and/or as a result of the population being subdivided into semi-isolated islands $\left(=F_{\mathrm{ST}}\right)$. The situation that these equations do not directly address, however, concerns how $N_{\mathrm{e}}$ is affected when genetic structure builds up as a result of 'isolation-bydistance'. Wright $(1943,1946)$ suggested how this could be done.

Wright (1946) introduced the concept of a neighborhood as the unit of structure that arises from isolation by distance within a continuously distributed population. He showed that when both sexes exhibit normally distributed dispersal (of either individuals or gametes), then $N_{\mathrm{n}}$ is the number of individuals contained in a circle of radius $2 \sigma$, that is,

$$
N_{\mathrm{n}}=4 \pi \sigma^{2} d
$$

where $\sigma^{2}$ is the variance of the distance between a central offspring and its parents, measured along a diameter (noting that the symmetry in the position of parents around the focal offspring defines a mean dispersal distance of zero), and where $d$ is the density of individuals. Note that Equation (3) indicates that $N_{\mathrm{n}}$ will be relatively constant assuming that $\sigma^{2}$ is a property of the species, but it will vary somewhat if the density of individuals varies among different populations. Even the effect of varying density may be minimized if there is some negative correlation between dispersal and density (for example, if at very low density, individuals disperse further).

The neighborhood number directly determines how random genetic drift affects genetic differentiation within a population, with a smaller neighborhood (because of a shorter dispersal distance) resulting in greater differentiation. Thus, within a large continuously distributed population, $N_{\mathrm{n}}$ provides information on the spatial distribution of genetic variation contained within a population. This genetic structure develops regardless of the total population size (assuming $N_{\mathrm{e}} » N_{\mathrm{n}}$ ). The value of $N_{\mathrm{n}}$ affects the level of genetic variation within the total population indirectly via the influence of this genetic structure that affects $N_{\mathrm{e}}$ by creating a pattern of nonrandom mating. As genetic structure created by limited dispersal is often a feature of natural populations, it is important to determine accurately what influence $N_{\mathrm{n}}$ has on the effective size of the total population.

Wright (1943) suggested that a random breeding unit in a continuous population (later called a neighborhood in Wright, 1946) was analogous to an island of the island model if neighborhoods were sampled at random. To formalize the analogy he showed that, if these units regulate their numbers locally, then:

$$
N_{\mathrm{e}}=N /\left(1-F_{\mathrm{ST}}\right)
$$

where $F_{\mathrm{ST}}$ is the genetic differentiation among randomly sampled neighborhoods. Equation (4) predicts that as the genetic structure becomes more extreme ( $F_{\mathrm{ST}}$ increases), then $N_{\mathrm{e}}$ increases.

Maruyama (1972) derived a more exact expression for the decline in heterozygosity given the isolation-by-distance model and using numerical examples of his formula concluded that:

$$
\begin{aligned}
& N_{\mathrm{e}}=N \quad \text { if } \quad \sigma^{2} d>1 \\
& N_{\mathrm{e}}=N /\left(\sigma^{2} d\right) \quad \text { if } \quad \sigma^{2} d<1
\end{aligned}
$$

From Equation (3), this result indicates that if $N_{\mathrm{n}}>4 \pi(=12.6)$, then neighborhood size has no noticeable effect on the global effective population size, but if the neighborhood size is small then:

$$
N_{\mathrm{e}}=4 \pi N / N_{\mathrm{n}}
$$

that is, $N_{\mathrm{e}}$ increases as $N_{\mathrm{n}}$ decreases, with $N_{\mathrm{e}}=4 \pi N$ when $N_{\mathrm{n}}=1$. This result is qualitatively (but not quantitatively) the same as Equation (4).

To further examine the link between $N_{\mathrm{n}}$ and $N_{\mathrm{e}}$, Kawata (1995) simulated a continuously distributed population where limited dispersal created genetic structure. During the initial nonequilibrium phase (he used the first 15 generations), while the genetic structure was being established, estimates of $N_{\mathrm{e}}$ (using the decline in heterozygosity) were closely related to $N_{\mathrm{n}}$, but later, when the genetic structure of the population had equilibrated, the estimates of $N_{\mathrm{e}}$ were more influenced by $N$. However, Kawata (1995) found that at these late stages the estimated $N_{\mathrm{e}}$ was always less than or equal to the population size, a result conflicting with Equation (4).

Neel et al. (2013) also simulated a neighborhood-structured population, estimating $N_{\mathrm{e}}$ with a single-sample estimator (based on linkage disequilibrium). Like Kawata (1995), they found that under some conditions (in their case, when the genetic sampling was at the scale of a single neighborhood) the estimated $N_{\mathrm{e}}$ was close to $N_{\mathrm{n}}$. Larger scale sampling encompassing several neighborhoods produced higher estimates of $N_{\mathrm{e}}$, but they never approached the expected theoretical value defined by Equation (4). For example, given the largest neighborhood size tested $(=84)$ and a sample area of $5 \%$ of a population of 90000 , the estimate of $N_{\mathrm{e}}$ was under 800 .

The present paper was motivated by questions arising from these two previous simulation studies. First, was Wright (1943) correct in his contention that the equilibrium interrelationship of $N_{\mathrm{e}}, N_{\mathrm{n}}$ and $N$ is closely approximated by the results derived for the island model, that is, that the effect of neighborhood structure on $N_{e}$ is analogous to the effect of island sub-populations on the effective size of the total population, as defined by Equation (4). The results of Kawata (1995) were inconsistent with this contention. Second, the two previous studies showed that, under some conditions in populations with significant spatial genetic structure, traditional estimators of $N_{\mathrm{e}}$ can lead to substantial underestimates closer to $N_{\mathrm{n}}$ than the expected $N_{\mathrm{e}}$. 
Furthermore, Neel et al. (2013) concluded that single-sample estimates of $N_{\mathrm{e}}$ will generally result in an underestimation of the true $N_{\mathrm{e}}$, and raised the question of whether this bias also applies to the more traditional two-sample temporal method (see Waples, 1989). If the temporal method underestimates the true $N_{\mathrm{e}}$ to a degree similar to that revealed by the simulation estimates of Neel et al. (2013) using a single-sample linkage disequilibrium method, then this might account for many of the unusually low estimates of $N_{\mathrm{e}} / N$ reported in the literature.

These two questions were investigated using simulations of a spatially structured plant population. First, to examine the accuracy of Equation (4), the 'true $N_{\mathrm{e}}$ ', that is, the value of $N_{\mathrm{e}}$ realized in a simulation over a period of 32 generations, was calculated from the gene frequency change occurring across 1000 single-nucleotide polymorphisms (SNPs) estimated from the total population. The reliability of this estimate was independently verified using $F_{\mathrm{ST}}$ values resulting from simulations of an island-structured metapopulation (of 1000 islands with 1 polymorphic locus), where each island showed internal genetic structure because of isolation by distance. Second, the bias in estimating $N_{\mathrm{e}}$ in a spatially structured population was evaluated using the temporal method under varying conditions of sample size, sampling method and time interval. Until recently, the temporal method was the main approach for estimating $N_{\mathrm{e}}$ using genetic data.

In summary, this paper addresses the theoretical problem of whether Equation (4) defines $N_{\mathrm{e}}$, and the practical problem of whether the temporal method can accurately estimate $N_{\mathrm{e}}$. Understanding the extent to which spatial genetic structure is expected to increase (or decrease) $N_{\mathrm{e}}$ is important in predicting the effect of factors such as habitat loss on the long-term genetic composition of populations using the theoretical links between ecological/demographic factors and $N_{\mathrm{e}}$ (Nunney and Elam, 1994). It is also important in evaluating genetic estimates of $N_{\mathrm{e}}$, given the concern over substantial bias noted by Neel et al. (2013). Documenting this bias across the available genetic estimators is the first step in moving toward a resolution of the problem.

\section{MATERIALS AND METHODS}

\section{Simulation model}

The simulation model assumed a plant population of monoecious annuals. The habitat consisted of a rectangular array of regularly spaced sites, each of which always supported a single plant so that the population size $N$ was constant. Pollen dispersal was normally distributed around each paternal plant and seed dispersal was zero; thus success through female function was fixed at one, whereas male success was approximately Poisson (although it was expected to be somewhat influenced by the neighborhood size). To avoid edge effects, it was assumed that locally dispersing pollen was reflected back from the boundaries of the population. The model was coded in PureBasic (Fantaisie Software, Fegersheim, France).

The simulated system tracked 1000 independent biallelic loci (or SNPs) initiated with equal allele frequencies. As noted above, the true value of $N_{\mathrm{e}}$ determining drift over a period of 32 generations was calculated using the temporal method by genetically sampling every individual before and after the 32-generation interval. This value was compared with shorter sampling intervals of $1,2,4,8$ and 16 generations. To examine the effect of less than complete sampling, three different sampling strategies were employed using intervals of $1,2,4,8,16$ and 32 generations. These strategies were: (1) random sampling over the whole population (without replacement), (2) sampling and resampling a single site and (3) sampling one site but resampling a different site. All sampling was nondestructive, and each sampling or resampling of a site included all individuals at that location. Sampling was initiated after an $N$-generation burn-in. The population sizes simulated were $N=256,1024$ or 4096 and the fraction of the population sampled was 25,10 or $2 \%$. If either one of an initial/final pair of samples was not polymorphic at a locus, then that locus was omitted as the time period over which drift was acting was unknown.

To estimate $N_{\mathrm{e}}$, the temporal method was applied using the statistic Fc and the appropriate sample size correction (Equations (8) and (12) from Waples, 1989). Each 1000-locus scenario of each of the three population sizes and each of the three sample sizes (that is, 9 cases) was replicated 5 times, with the three sampling methods being simultaneously implemented within each simulation.

The expected value of $N_{\mathrm{e}}$ given uniform pollen dispersal (that is, $N_{\mathrm{n}}=\infty$ ) was used as a reference value $\left(N_{e, r e f}\right)$ for testing the fit of the data to theory. $N_{\text {e,ref }}$ was predicted to be $4 N / 3$ based on Wright's (1938) classic result of $N_{\mathrm{e}}=4 N /(2+V)$, noting that the reproductive variance $V$ is made up of male plus female variance. Given the conditions of the simulation, $V_{\mathrm{f}}=0$ whereas $V_{\mathrm{m}} \approx 1$.

To provide an independent estimate of $N_{\mathrm{e}}$ given the conditions of the simulation, $N_{\mathrm{e}}$ was also estimated from the equilibrium $F_{\mathrm{ST}}$ using simulations of 1000 neighborhood-structured island populations (each of $N=256,1024$ or 4096) linked by a low level of migration $\left(N_{\mathrm{m}}=1\right)$ and segregating a single biallelic locus, so that $N_{\mathrm{e}}=\left(1-F_{\mathrm{ST}}\right) /\left(4 m F_{\mathrm{ST}}\right)$. Simulations were run for $4 N$ generations, and estimates of $F_{\mathrm{ST}}$ were based on the final $N$ generations.

\section{RESULTS}

\section{The effect of $N_{\mathrm{n}}$ on $N_{\mathrm{e}}$}

When the neighborhood size was infinite (that is, uniform pollen dispersal), the effective size determined from gene frequency change occurring over 32 generations $\left(N_{\mathrm{e}, 32}\right)$ was in close agreement with the expected value of $4 N / 3\left(=N_{\mathrm{e}, \mathrm{ref}}\right): N_{\mathrm{e}, 32}=360 \pm 15$ (mean \pm 1 s.d.) with $N_{\mathrm{e}, \text { ref }}=341.3 \quad(N=256) ; \quad N_{\mathrm{e}, 32}=1396 \pm 78$ with $N_{\mathrm{e}, \text { ref }}=1365$ $(N=1024)$; and $N_{e, 32}=5526 \pm 230$ with $N_{e, \text { ref }}=5461 \quad(N=4096)$, where each value was based on 15 replicates. Under these conditions, genetic structure was minimal $\left(F_{I S}=0.00\right)$, and, as expected, when neighborhood size was reduced (using a series of fourfold reductions down to $N_{\mathrm{n}}=1$ ), genetic structure increased, as measured by $F_{\text {IS }}$ (Table 1). Determining $N_{\mathrm{e}}$ from the gene frequency change occurring over 32 generations $\left(N_{e, 32}\right)$, it was found that reducing $N_{\mathrm{n}}$ increased $N_{\mathrm{e}}$, although the effect was relatively minor until the neighborhood size was very small (roughly less than 16; Figure 1). This increase was compared with the expected effective size $\left(N_{\mathrm{e}, \text { exp }}\right)$ calculated as:

$$
N_{e, \exp }=N_{e, \text { ref }} /\left(1-F_{\mathrm{IS}}\right)
$$

Using Equation (7) provides a practical test of the suggestion of Wright (1943) that the isolation-by-distance model should exhibit the same genetic structure as the island model among neighborhoods. As neighborhood dimensions cannot be easily identified in the field, an alternative to comparing neighborhoods is to quantify genetic structure using the population-wide $F_{\text {IS. The results showed that }}$ Equation (7) generally provides a good estimate of $N_{\mathrm{e}}$ (Figure 1). It did overestimate the effective size somewhat when $N_{\mathrm{n}}$ was very small $(\leqslant 4)$. Thus for $N_{\mathrm{n}}=4$, the bias was limited to $7 \%$, but increased to $\sim 20 \%$ when $N_{\mathrm{n}}=1$ ( $18 \%$ when $N=256$ and increasing to $23 \%$ when $N=4096)$.

The $N_{\mathrm{e}}$ based on the population-wide gene frequency changes observed across 32 generations $\left(N_{\mathrm{e}, 32}\right)$ were compared with independent estimates derived from a separate set of simulations in which 1000 replicate populations were linked by random dispersal $\left(N_{\mathrm{m}}=1\right)$. $N_{\mathrm{e}}$ was calculated from $F_{\mathrm{ST}}$ among the island populations. The results are shown in Table 1 and are very similar to the temporal method values, except when $N_{\mathrm{n}}$ is very small. For $N_{n} \leqslant 4$, the $F_{\mathrm{ST}}$-based estimates were significantly lower, an effect apparently linked to reduced genetic structure within the populations (that is, reduced $F_{\text {IS }}$; see Table 1) because of immigration. 

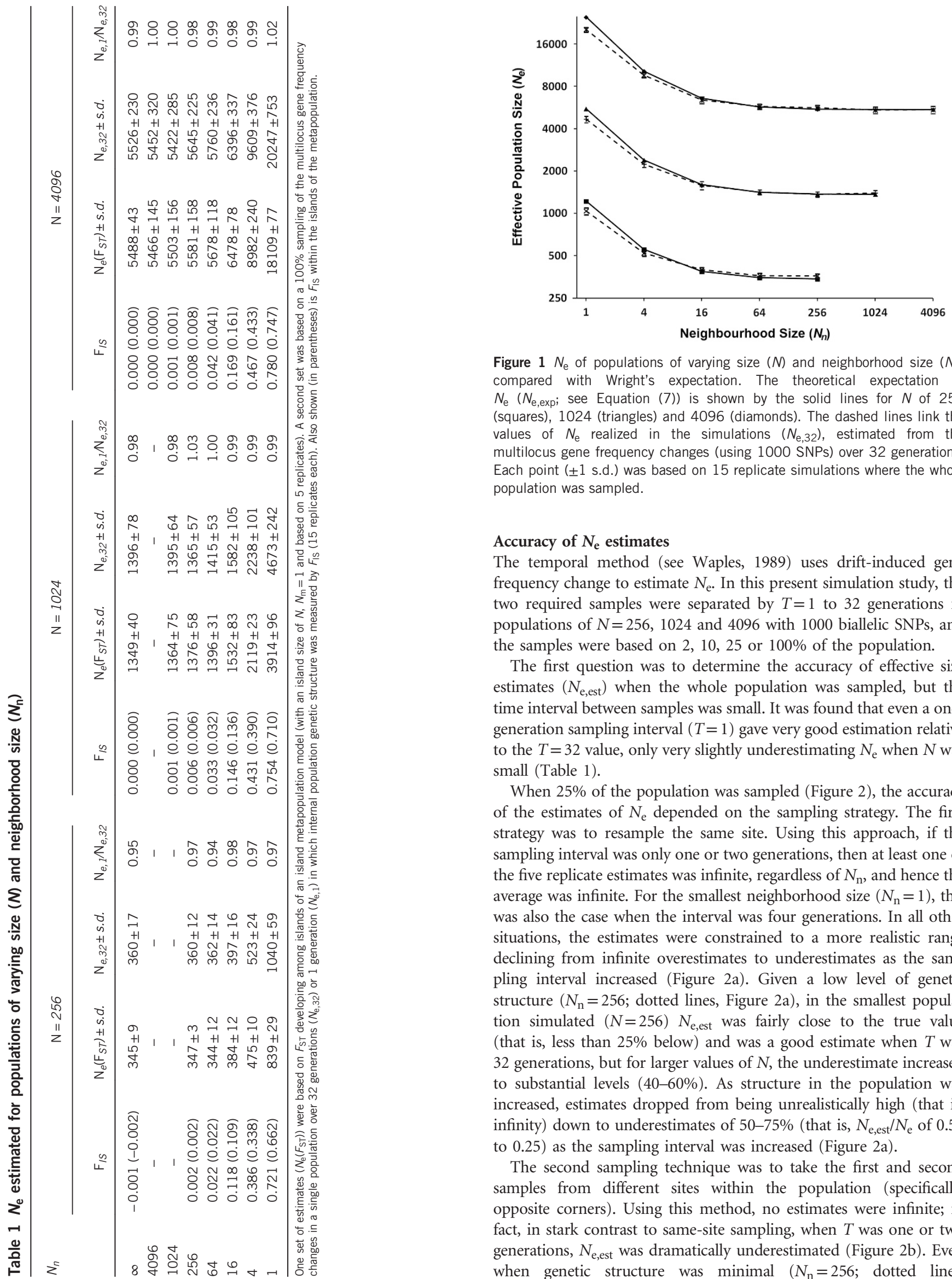

Figure $1 N_{\mathrm{e}}$ of populations of varying size $(N)$ and neighborhood size $\left(N_{n}\right)$ compared with Wright's expectation. The theoretical expectation of $N_{\mathrm{e}}\left(N_{\mathrm{e}, \text { exp }}\right.$; see Equation (7)) is shown by the solid lines for $N$ of 256 (squares), 1024 (triangles) and 4096 (diamonds). The dashed lines link the values of $N_{\mathrm{e}}$ realized in the simulations $\left(N_{\mathrm{e}, 32}\right)$, estimated from the multilocus gene frequency changes (using 1000 SNPs) over 32 generations. Each point $( \pm 1$ s.d.) was based on 15 replicate simulations where the whole population was sampled.

\section{Accuracy of $N_{\mathrm{e}}$ estimates}

The temporal method (see Waples, 1989) uses drift-induced gene frequency change to estimate $N_{\mathrm{e}}$. In this present simulation study, the two required samples were separated by $T=1$ to 32 generations in populations of $N=256,1024$ and 4096 with 1000 biallelic SNPs, and the samples were based on 2, 10, 25 or $100 \%$ of the population.

The first question was to determine the accuracy of effective size estimates $\left(N_{\mathrm{e}, \text { est }}\right)$ when the whole population was sampled, but the time interval between samples was small. It was found that even a onegeneration sampling interval $(T=1)$ gave very good estimation relative to the $T=32$ value, only very slightly underestimating $N_{\mathrm{e}}$ when $N$ was small (Table 1).

When $25 \%$ of the population was sampled (Figure 2), the accuracy of the estimates of $N_{\mathrm{e}}$ depended on the sampling strategy. The first strategy was to resample the same site. Using this approach, if the sampling interval was only one or two generations, then at least one of the five replicate estimates was infinite, regardless of $N_{\mathrm{n}}$, and hence the average was infinite. For the smallest neighborhood size $\left(N_{n}=1\right)$, this was also the case when the interval was four generations. In all other situations, the estimates were constrained to a more realistic range declining from infinite overestimates to underestimates as the sampling interval increased (Figure 2a). Given a low level of genetic structure $\left(N_{\mathrm{n}}=256\right.$; dotted lines, Figure 2a), in the smallest population simulated $(N=256) N_{e, \text { est }}$ was fairly close to the true value (that is, less than $25 \%$ below) and was a good estimate when $T$ was 32 generations, but for larger values of $N$, the underestimate increased to substantial levels $(40-60 \%)$. As structure in the population was increased, estimates dropped from being unrealistically high (that is, infinity) down to underestimates of $50-75 \%$ (that is, $N_{\mathrm{e}, \text { est }} / N_{\mathrm{e}}$ of 0.50 to 0.25 ) as the sampling interval was increased (Figure 2a).

The second sampling technique was to take the first and second samples from different sites within the population (specifically, opposite corners). Using this method, no estimates were infinite; in fact, in stark contrast to same-site sampling, when $T$ was one or two generations, $N_{e, \text { est }}$ was dramatically underestimated (Figure $2 \mathrm{~b}$ ). Even when genetic structure was minimal $\left(N_{\mathrm{n}}=256\right.$; dotted lines, 
Figure 2b), the underestimate was such that $N_{\text {e,est }} / N_{\mathrm{e}}$ was 0.12 $(N=256)$ to $0.008(N=4096)$ when $T=1$. The underestimate became more extreme as the neighborhood size decreased. For $N_{\mathrm{n}}=1$, the
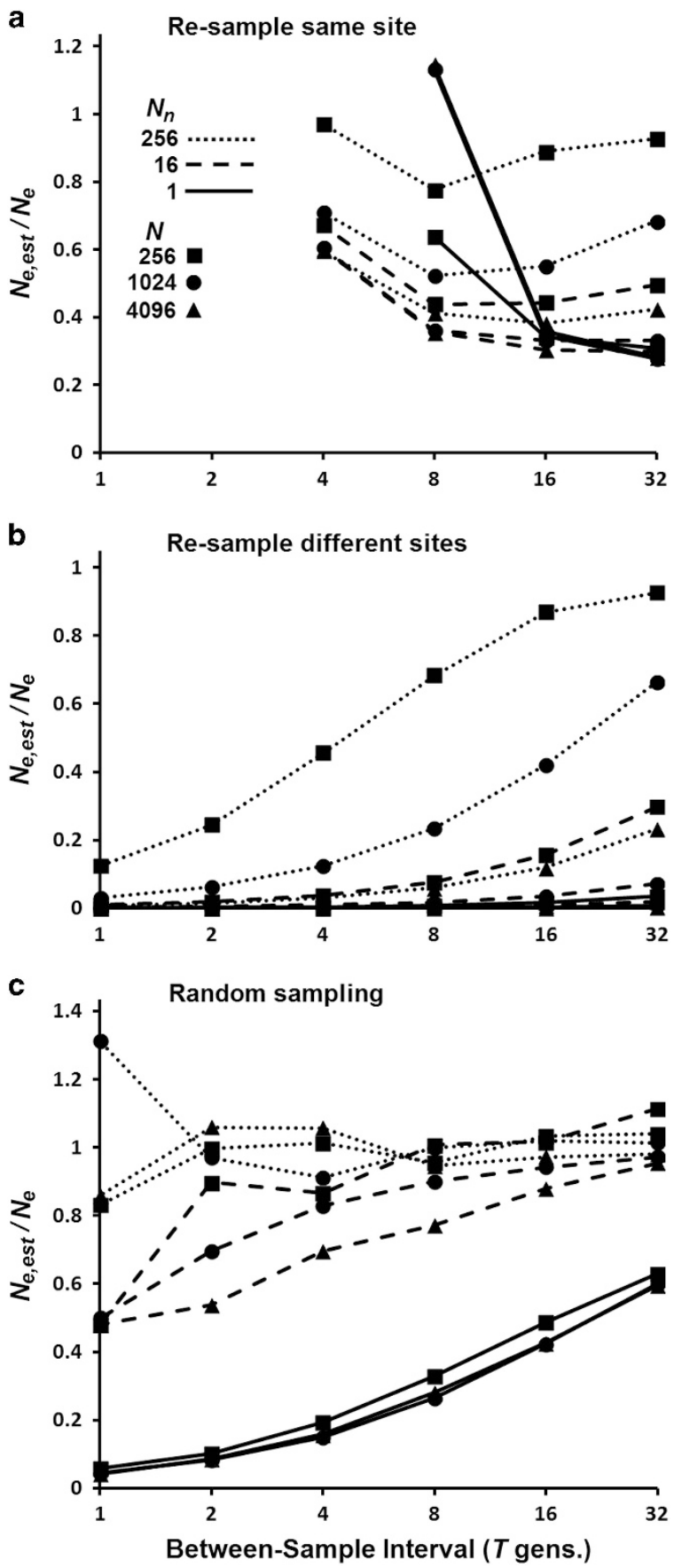

Figure 2 The accuracy of temporal-method estimates of $N_{e}$ based on sampling $25 \%$ of the population after various intervals $(T)$. The estimate of $N_{\mathrm{e}}$ is shown relative to $N_{\mathrm{e}, 32}$, the value derived from sampling the whole population over a period of 32 generations (see Figure 1). Three sampling methods are illustrated: (a) resample all individuals in the same site; (b) sample all individuals in one site and then resample all individuals from a non-overlapping site; and (c) randomly sample individuals for both samples. The results are shown for different populations sizes $(N=256,1024$ and 4096) and neighborhood sizes $\left(N_{n}=1,16\right.$ and 256). Five sets of simulations with 1000 loci were run for $N$ generations. Points that included infinite estimates of $N_{\mathrm{e}}$ were omitted. ratio of $\mathrm{N}_{\mathrm{e}, \mathrm{est}} / \mathrm{N}_{\mathrm{e}}$ was $0.001(N=256)$ to $0.0001 \quad(N=4096)$ given $T=1$, a situation that improved as $T$ increased, but the underestimate remained extreme even for $T=32$ (when the equivalent ratios were 0.04 to 0.002 ).

The third sampling technique, random sampling across the whole population, was generally the most accurate of the three methods. This accuracy was notable when $N_{\mathrm{n}}=256$ (dotted lines, Figure 2c); however, especially when the sampling interval was small $(T=1$ or 2 ), there was an increasing downward bias as genetic structure increased. Thus when $N_{\mathrm{n}}=1$ (solid lines, Figure 2c), $N_{\mathrm{e}, \text { est }}$ was $\sim 5 \%$ of $N_{\mathrm{e}}$ when $T=1$ and $\sim 10 \%$ when $T=2$.

Reducing the sample size to 10 or $2 \%$ of the total population had qualitatively the same effect on the estimate of $N_{\mathrm{e}}$ as a $25 \%$ sample; however, quantitatively the estimates of $N_{\mathrm{e}}$ became smaller as the sample size decreased. Figure 3 shows the pattern for $N=1024$. The only exceptions to this pattern were estimates from smaller unstructured populations, some of which became infinite. For example, given same-site sampling, the estimates that were infinite given $25 \%$ sampling, remained so given 10 and $2 \%$ sampling, but others were added under $2 \%$ sampling. For example, when $N_{\mathrm{n}}=256$, average estimates were infinite over a broader range of $T$ : for $T \leqslant 16$ when $N=256$; for $T \leqslant 8$ when $N=1024$; and for $T \leqslant 4$ when $N=4096$. Under the same conditions ( $2 \%$ sampling and $N_{\mathrm{n}}=256$ ), infinite estimates were also seen with random sampling (for $T \leqslant 16$ when $N=256$; for $\mathrm{T} \leqslant 8$ when $N=1024$; and for $T \leqslant 2$ when $N=4096$ ), whereas different-site resampling only gave infinite estimates when $N=256$ (for $T \leqslant 16$ ).

The degree of underestimate given 10 and $2 \%$ sampling is further quantified in Table 2 for $N_{\mathrm{n}}=16$, a case of moderate spatial structure $\left(0.1<F_{\text {IS }}<0.2\right.$; see Table 1$)$, that one would hope would not be too much of a challenge for estimating $N_{\mathrm{e}}$. However, this was not the case. Although the estimates were consistent (that is, a low coefficient of variation), the accuracy was generally poor, and often very poor. Given different-site resampling (Figure 3b), the estimates were often more than two orders of magnitude in error, an effect that was most pronounced when the population was large (underlined values in Table $2 \mathrm{~b}$ ). The accuracy improved when the time period between generations was long (for example, $T=32$ generations), but the estimates were still very biased. Given same-site resampling, the underestimation was reduced relative to the case of different-site resampling (Table 2a); however, the underestimate was still $\sim 3-30$-fold (dashed and solid lines, Figure 3a). As noted earlier, the pattern of underestimation was reversed if the sample interval was only one or two generations when $N_{e}$ was overestimated, generally as infinite (Figure 3a).

In contrast, random sampling across the whole population gave markedly better estimates (Figure 3c). The accuracy was generally good across all sampling proportions when $T=32$ generations, except when the genetic structure was extreme $\left(N_{\mathrm{n}}=1\right.$; see triangles, Figure 3c). Accuracy deteriorated as the time interval between samples was shortened, but the underestimation was modest compared with the other methods (Table 2c), provided the neighborhood size was not too small (Figure 3c); however, as $T$ was reduced, the estimator could flip from significant underestimation to infinite overestimation (Table 2c and Figure 2c).

\section{DISCUSSION}

The work presented was designed to emphasize the important distinction between the effective size of a population $\left(N_{\mathrm{e}}\right)$ and its internal neighborhood size $\left(N_{\mathrm{n}}\right)$. Random genetic change at the population level is determined by $N_{\mathrm{e}}$ that is largely dependent 

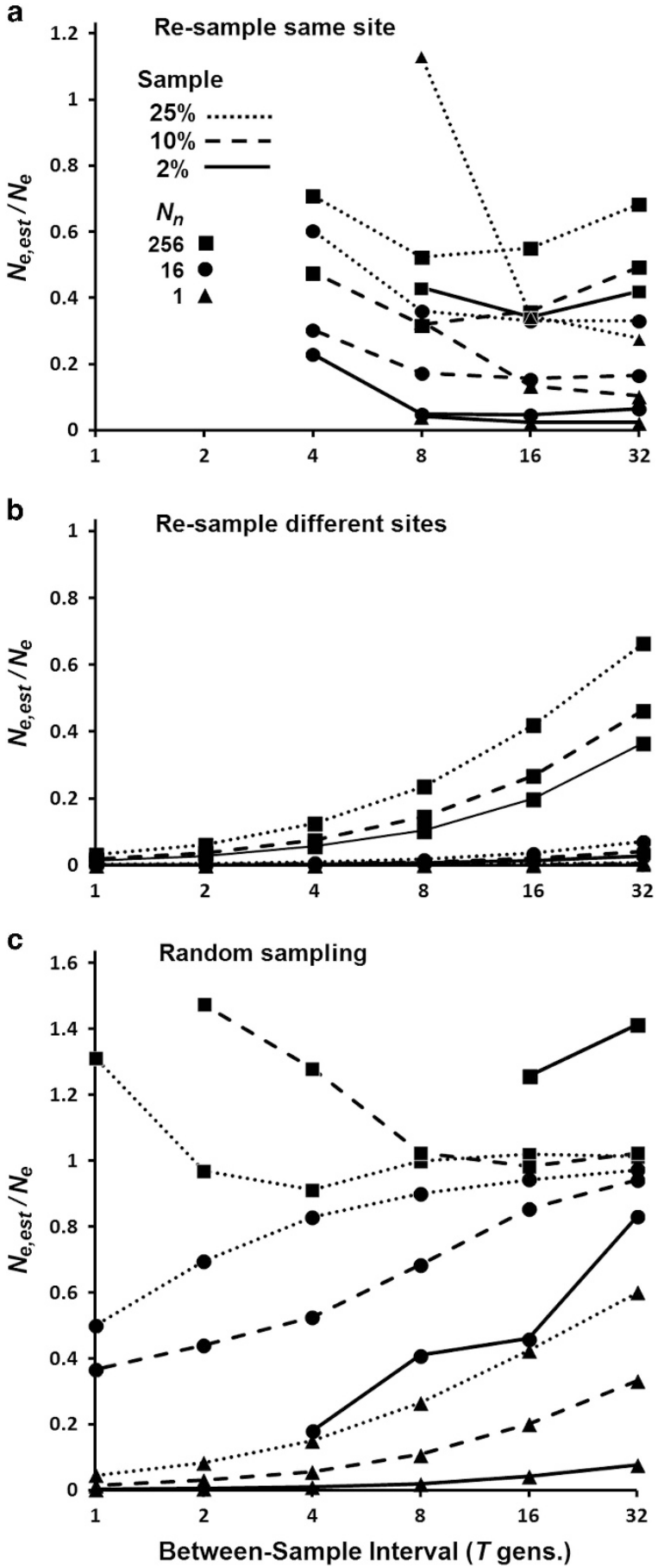

Figure 3 The effect of sampling fraction on the accuracy of temporalmethod estimates of $N_{\mathrm{e}}$ given the three different sampling strategies of (a) resampling the same site, (b) resampling a different site or (c) random sampling. The fraction of the population $(N=1024)$ sampled was 25,10 or $2 \%$. Details are as described in Figure 2.

upon $N$, the number of adults in the population, that in turn is largely determined by the area of the suitable habitat. It is $N_{\mathrm{e}}$ that determines the long-term effects of genetic drift on the genetic composition of a population. In contrast, $N_{\mathrm{n}}$ is primarily dependent upon the dispersal patterns of the species (see Equation (3)), and provides no direct information on the fate of genetic variation in the population as a whole.
In a population structured by the influence of isolation by distance, neighborhoods are, to some degree, genetically differentiated from each other; however, the genetic composition of each neighborhood also varies over time. In essence, given spatial structure, gene frequency contours drift over time, even in a large population where the overall gene frequency remains largely unchanged. This spatial drift has important consequences for the estimation of $N_{\mathrm{e}}$ (see below).

It is important that the distinction between these two measures is kept clear, but the terminology can sometimes be confusing. For example, $N_{\mathrm{n}}$ is sometimes called the neighborhood or local effective population size (see, for example, Eguiarte et al., 1993; Neel et al., 2013), and the neighborhood size is sometimes symbolized by $N_{\mathrm{e}}$ (Kawata, 1995). This can lead others to the incorrect assumption that $N_{\mathrm{n}}$ exhibits the properties of $N_{\mathrm{e}}$ that are important in maintaining genetic variation in a population. For example, Lode and Peltier (2005) in their study of mink concluded that their estimate of $N_{n}$ (of 16-23) was far below values considered critical for long-term viability. This was not an appropriate conclusion; they were comparing estimates of $N_{\mathrm{n}}$ with a suggested theoretical minimum applying to $N_{\mathrm{e}}$. Under most circumstances, and especially in the context of populations at risk for extinction, $N_{\mathrm{e}}$ is the critical parameter determining the long-term maintenance of genetic variation (see Nunney, 2000). The magnitude of $N_{\mathrm{n}}$ is only relevant in such studies to the extent that $N_{\mathrm{n}}$ influences $N_{\mathrm{e}}$.

However, $N_{\mathrm{n}}$ does have an indirect effect on the level of genetic variation by its influence on $N_{\mathrm{e}}$. Wright (1943) predicted that a continuous population structured by limited dispersal would behave much like a population consisting of a set of island sub-populations. The link between such 'isolation-by-distance' populations and a system with separate island sub-populations was supported by Slatkin and Barton (1989), who noted that the stepping stone and neighborhood models can be equated using $N_{\mathrm{n}}=2 \pi n m$, where $n$ is the size of a sub-population and $m$ is the migration rate among adjacent sub-populations in a stepping-stone model.

The simulations of annual plant populations presented here supported this view: neighborhood size $\left(N_{\mathrm{n}}\right)$ affected the effective size of the population $\left(N_{\mathrm{e}}\right)$ in a manner consistent with Equation (7), a simple reformulation of the island model Equation (4). The only notable deviation occurred when $N_{\mathrm{n}}$ was very small (=1; see Figure 1). In any event, as $N_{\mathrm{n}}$ decreased, causing genetic structure to become more pronounced, $N_{\mathrm{e}}$ increased. This is to be expected, because as neighborhoods become increasingly different from each other, local structure protects genetic variation from being lost from the population by drift, provided population regulation is local (see below). The effect of $N_{\mathrm{n}}$ on $N_{\mathrm{e}}$ is only apparent when the neighborhood size is $<16$ when $N=4096$ (when $F_{\text {IS }}=0.16$; see Table 1), and when $N_{\mathrm{n}}$ is a little smaller for smaller $N$ (Figure 1). This result is in general agreement with the conclusion of Maruyama (1972), who suggested that $N_{\mathrm{n}}$ had little effect on $N_{\mathrm{e}}$ if $\sigma^{2} d>1$ that, as noted earlier, translates to $N_{\mathrm{n}}>12.6$; however, the results did not fit well with the prediction of Maruyama (1972) (Equation (6)) for $N_{\mathrm{e}}$ when $N_{\mathrm{n}}$ is small. For example, when $N_{\mathrm{n}}=1$, the prediction is that $N_{\mathrm{e}}$ is increased 16.7-fold by genetic structure, whereas the simulations gave much lower values, between $2.9(N=256)$ and $3.7(N=4096)$ (Table 1).

In contrast to these patterns, Kawata (1995) found, in a series of simulations, that $N_{\mathrm{e}}$ decreased when $N_{\mathrm{n}}$ was reduced. Although the reasons for this apparently contradictory result cannot be identified with certainty, the simulations are consistent with a decrease in $N_{\mathrm{e}}$ because of two factors: the mating system and global population regulation. 
Table 2 The bias in effective size estimates $\left(N_{\mathrm{e}, \text { est }}\right)$ given a neighborhood size $\left(N_{n}\right)$ of 16

\begin{tabular}{|c|c|c|c|c|c|c|c|c|c|c|}
\hline \multirow[t]{2}{*}{ Sample\% } & \multicolumn{4}{|c|}{$N=256$} & \multicolumn{3}{|c|}{1024} & \multicolumn{3}{|c|}{4096} \\
\hline & T & $\mathrm{N}_{e, e s t}$ & $\mathrm{CV}$ & $\mathrm{N}_{e, e s t} / \mathrm{N}_{e, 32}$ & $\mathrm{~N}_{e, e s t}$ & CV & $\mathrm{N}_{e, e s t} / \mathrm{N}_{e, 32}$ & $\mathrm{~N}_{e, \text { est }}$ & CV & $\mathrm{N}_{e, \text { est }} / \mathrm{N}_{e, 32}$ \\
\hline \multicolumn{11}{|c|}{ (a) Same-site resampling } \\
\hline \multirow[t]{3}{*}{10} & 32 & 116 & 0.055 & 0.2912 & 261 & 0.060 & 0.1698 & 875 & 0.058 & 0.1372 \\
\hline & 8 & 82 & 0.133 & 0.2050 & 272 & 0.053 & 0.1771 & 1023 & 0.064 & 0.1603 \\
\hline & 2 & $\infty$ & & & $\infty$ & & & $\infty$ & & \\
\hline 2 & 2 & $\infty$ & & & $\infty$ & & & $\infty$ & & \\
\hline \multicolumn{11}{|c|}{ (b) Different-site resampling } \\
\hline \multirow[t]{2}{*}{10} & 32 & 68 & 0.029 & 0.1712 & 65 & 0.033 & 0.0427 & 68 & 0.072 & 0.0107 \\
\hline & 8 & 17 & 0.069 & 0.0423 & 16 & 0.049 & 0.0105 & 17 & 0.070 & $\underline{0.0027}$ \\
\hline \multicolumn{11}{|c|}{ (c) Random sampling } \\
\hline \multirow[t]{3}{*}{10} & 32 & 383 & 0.068 & 0.9597 & 1475 & 0.061 & 0.9609 & 6174 & 0.088 & 0.9680 \\
\hline & 8 & 299 & 0.045 & 0.7505 & 1072 & 0.211 & 0.6982 & 4176 & 0.117 & 0.6547 \\
\hline & 2 & 169 & 0.307 & 0.4227 & 691 & 0.284 & 0.4501 & 1750 & 0.154 & 0.2743 \\
\hline \multirow[t]{3}{*}{2} & 32 & 549 & 0.287 & 1.3902 & 1302 & 0.198 & 0.7922 & 3555 & 0.099 & 0.5539 \\
\hline & 8 & 409 & 0.806 & 1.0363 & 642 & 0.588 & 0.3905 & 1806 & 0.193 & 0.2813 \\
\hline & 2 & $\infty$ & & & $\infty$ & & & 639 & 0.206 & 0.0996 \\
\hline
\end{tabular}

The size of the two samples was 10 or $2 \%$ of the total population $(N=256,1024$ or 4096$)$, taken $T$ generations apart. The values shown are $N_{\text {e,est, }}$ its coefficient of variation (CV) based on 5 estimates and the ratio of $N_{\mathrm{e}, e s t}$ to the estimate of $N_{\mathrm{e}}$ based on sampling the whole population with $T=32\left(N_{\mathrm{e}, 32}\right)$. Estimates of $N_{\mathrm{e}, \text { est }} / N_{\mathrm{e}, 32}$ in bold are underestimates of at least $10-$ fold, and those that are also underlined are $>100$-fold underestimates.

In his simulations, individuals were non-selfing hermaphrodites. Female parents were chosen randomly (with replacement) from across the population and for each a male parent was chosen within a circle of radius $M$. When $M$ is large, this mating system approximates a random union of gametes model; however, when $M$ is small the system becomes more complex. For example, some individuals have zero fitness because they do not have any potential mates within the specified area, thus increasing the overall variance in reproductive success and decreasing $N_{\mathrm{e}}$ (Kawata, 1995).

The effect of $M$ on the mating system accounted for some of the reduction in $N_{\mathrm{e}}$ below $N$ (see figure 6b of Kawata, 1995), but the effect was not strong enough to explain why reducing $N_{\mathrm{n}}$ showed no indication of driving the increase in $N_{\mathrm{e}}$ predicted by Equation (4). The primary reason why $N_{\mathrm{n}}$ reduced rather than increased $N_{\mathrm{e}}$ appears to be because of the nature of the population regulation.

Kawata (1995) implicitly assumed that population regulation acted globally, that is, limiting the whole population to size $N$, whereas the simulations presented here imposed strong local regulation (one individual at each of the $N$ sites). Given local regulation at or below the level of the neighborhood makes the isolation-by-distance model analogous to the island model (as pointed out by Wright, 1943); however, global regulation changes the model to one that is more analogous to a demic model (Nunney, 1999). In the island model, shifting from local (island level) to global (population level) regulation of dispersal reverses the effect of $F_{\mathrm{ST}}$ on $N_{\mathrm{e}}$ (compare Equations (1) and (2)). Similarly, given isolation by distance, global population regulation results in random neighborhood productivity differences driving increased genetic drift and hence lowering $N_{\mathrm{e}}$.
Whether population regulation is local or global will depend on the specific factors acting; however, many density-dependent factors (notably intraspecific and interspecific competition) act locally, suggesting that regulation may generally act at the neighborhood level.

Estimates of $N_{\mathrm{e}}$ based on both the temporal method and using $F_{\mathrm{ST}}$ from independent simulations of a metapopulation of replicate populations (islands) linked by dispersal were highly concordant, except when $N_{\mathrm{n}}$ was very small $(\leqslant 4)$. This divergence was most probably because of the effect of immigration in reducing the internal genetic structure (that is, reducing $F_{I S}$, see Table 1 ). It is expected that reduced $F_{\text {IS }}$ would result in reduced $N_{\mathrm{e}}$ (see Equation (7)), and this is what is observed.

The presence of genetic spatial structure has been shown to strongly bias estimates based on the single-sample linkage disequilibrium method for the estimation of $N_{\mathrm{e}}$. Neel et al. (2013) found that estimates of $N_{\mathrm{e}}$ were close to $N_{\mathrm{n}}$ when the sample area was small relative to the neighborhood, and increased only slowly as the sample area increased, never approaching the true value of $N_{\mathrm{e}}$. In the present study, this pattern was only weakly supported given the comparable approach of same-site sampling, and required $T$ to be large enough to avoid infinite estimates (see Figures $2 \mathrm{a}$ and $3 \mathrm{a}$ ). For example, when $T=32$ and $N=1024$, the $2 \%$ sample size was well within a single neighborhood given $N_{\mathrm{n}}=64$ and 256, but $N_{\text {e,est }}$ was 201 and 578 respectively, values substantially larger than $N_{\mathrm{n}}$, although substantially smaller than $N_{\mathrm{e}}$ (estimated at 1415 and 1365, respectively, based on sampling the entire population).

The general conclusion regarding the two-sample temporal method for estimating $N_{\mathrm{e}}$ must be that the results cannot be trusted. The only conditions yielding relatively accurate results were random sampling 
of a large fraction $(\sim 25 \%)$ of a population with moderate to low structure $\left(F_{I S}<0.2\right)$ across an interval of $\geqslant 8$ generations (Figure $2 c$ ). If the proportion of the population sampled drops and/or there is significant spatial structure then accuracy quickly declines, an effect exacerbated by a reduced interval between samples (Figure $3 c$ and Table $2 \mathrm{c}$ ). In general, the values obtained with random sampling were underestimates, but when $N_{\mathrm{n}}$ was large and the sample size was small, they were overestimates. For example, it can be seen in Figure 3c (where $N=1024$ throughout) that when the sample size was $2 \%$, the estimates for $N_{\mathrm{n}}=256$ were slight overestimates for $T=16$ or 32 generations, but for shorter intervals $(T \leqslant 8)$ the estimates were infinite.

Same-site sampling yields dramatic (generally infinite) overestimates of $N_{\mathrm{e}}$ when $T$ is small, and underestimates when $T$ is larger. These underestimates can become substantial (that is, an order of magnitude or more) if the neighborhood size is small and the sample is taken from an area that represents a small fraction of the population (Figure 3a and Table 2a). It is important to understand the cause of this switch from extreme overestimation to underestimation as $T$ is increased. When $T$ is small, it is clear that limited (local) dispersal has the effect of buffering genetic change so that sampling the same site will result in less genetic change than is occurring in the population as a whole. This results in infinite estimates of $N_{\mathrm{e}}$. However as the time interval increases, the contours of gene frequency within the population shift in space, so that the change in gene frequency at a particular site is a combination of both population-wide drift and these local changes in gene frequency. The result is that $N_{\mathrm{e}}$ is underestimated.

The effect of internal genetic structure is even more apparent when different-site resampling is employed. It is clear that unless the time interval between samples is substantial, the gene frequency difference between the initial and final samples includes both the effect of population-wide drift and the effect of gene frequency differences between the two locations. This inevitably results in a very low estimate of $N_{\mathrm{e}}$ (see Figures $2 \mathrm{~b}$ and $3 \mathrm{~b}$ ). It was shown that these low estimates were often two or more orders of magnitude below the correct value, especially when $T$ was small and $N$ large (Table $2 b$ ).

The results presented were from simulations with varying levels of pollen dispersal but zero seed dispersal. This scenario may create particular difficulties for the estimation methods; however, the general pattern of very limited gene flow in one sex is neither uncommon nor restricted to plants. In animal species, it is frequently the case that one sex is philopatric, whereas the other disperses. Furthermore, the enormous bias observed in the estimates of $N_{\mathrm{e}}$ were also apparent in the results of Neel et al. (2013) given two-sex dispersal. It is clear that a new approach to the estimation of $N_{\mathrm{e}}$ in spatially structured populations is needed.

An important question raised by Neel et al. (2013) was whether isolation by distance could contribute to the unexpectedly low $N_{\mathrm{e}} / N$ ratios sometimes observed. At first sight this may appear paradoxical given that, under conditions of local population regulation, the simulations showed how a small $N_{\mathrm{n}}$ increases the true $N_{\mathrm{e}}$ in accord with Equation (4) (see Figure 1); however, in contrast to the effect of $N_{\text {n }}$ on the true $N_{\mathrm{e}}$, it is apparent that a small $N_{\mathrm{n}}$ can lead to estimates of $N_{\mathrm{e}}$ that dramatically underestimate the true $N_{\mathrm{e}}$ (Table 2).

Theory suggests that in general $N_{\mathrm{e}} / N \geqslant 0.1$ (Nunney and Campbell, 1993; Frankham, 1995; Vucetich et al., 1997), and although empirical evidence is broadly supportive of this conclusion (Palstra and Ruzzante, 2008), some exceptionally low ratios in the range $10^{-3}$ $10^{-5}$ have been published, many of which are derived from marine populations (reviewed in Hare et al., 2011). These studies typically used samples stored by previous researchers and, as such, may well result in resampling in a location that was different from the original. In addition, the populations are generally very large. It is perhaps notable that in the simulations these two factors resulted in the highest degree of underestimation (see Table 2).

The effective population size $N_{\mathrm{e}}$ is a very important population parameter for understanding long-term genetic change, whereas the neighborhood size $N_{\mathrm{n}}$ provides very different information concerning the degree to which the standing genetic variation becomes spatially structured. $N_{\mathrm{e}}$ is especially important in the context of predicting genetic loss from small populations of threatened species (Nunney, 2000), but it is also important for understanding genetic change in larger populations of commercially important species, notably fish (Hare et al., 2011). For these reasons accurate estimation of $N_{\mathrm{e}}$ is important.

The present work using the temporal method builds on the results of Neel et al. (2013) using the linkage disequilibrium method to demonstrate that current procedures for estimating $N_{\mathrm{e}}$ are woefully inaccurate in populations exhibiting spatial genetic structure. Strong biases were apparent even though the genetic data (based on 1000 SNPs) were extensive. Further theoretical work is urgently needed to resolve the confounding effect of spatial variation in the estimation of $N_{\mathrm{e}}$.

\section{DATA ARCHIVING}

Summary data from all simulation runs and the code used in this study are available from the Dryad Digital Repository: DOI:10.5061/dryad.qc1nc.

\section{CONFLICT OF INTEREST}

The author declares no conflict of interest.

\section{ACKNOWLEDGEMENTS}

I thank Martin Husemann, Frank Zachos, Robert Paxton, and Jan Habel for stimulating me to complete this research. I also thank the reviewers for their very thoughtful suggestions.

Caballero A, Hill WG (1992). Effective size of nonrandom mating populations. Genetics 130: 909-916.

Eguiarte LE, Burquez A, Rodriguez J, Martinez-Ramos M, Sarukhan J, Pinero D (1993). Direct and indirect estimates of neighborhood and effective population size in a tropical palm, Astrocaryum mexicanum. Evolution 47: 75-87.

Frankham R (1995). Effective population size adult population size ratios in wildlife: a review. Genet Res 66: 95-107.

Gilpin M (1991). The genetic effective size of a metapopulation. Biol J Linn Soc 42 $165-175$.

Hare M, Nunney L, Schwartz M, Ruzzante D, Burford M, Waples R et al. (2011). Understanding and estimating effective population size for practical application in marine species management. Cons Biol 25: 438-449.

Hedrick PW, Gilpin ME (1996). Genetic effective size of a metapopulation. In: Hanski IA, Gilpin ME (eds) Metapopulation Dynamics: Ecology, Genetics, and Evolution. Academic Press: London. pp 165-181.

Kawata M (1995). Effective population size in a continuously distributed population. Evolution 49: 1046-1054.

Lode T, Peltier D (2005). Genetic neighbourhood and effective population size in the endangered European mink Mustela lutreola. Biodiversity and Conservation 14: 251-259.

Maruyama T (1972). Rate of decrease of genetic variability in a two-dimensional continuous population of finite size. Genetics 70: 639-651.

Neel MC, McKelvey K, Ryman N, Lloyd MW, Short Bull R, Allendorf FW et al. (2013). Estimation of effective population size in continuously distributed populations: there goes the neighborhood. Heredity 111: 189-199.

Nunney L (1993). The influence of mating system and overlapping generations on effective population size. Evolution 47: 1329-1341.

Nunney L (1999). The effective size of a hierarchically-structured population. Evolution 53 : $1-10$.

Nunney L (2000). The limits to knowledge in conservation genetics: the value of effective population size. Evol Biol 32: 179-194.

Nunney L, Campbell KA (1993). Assessing minimum viable population size: demography meets population genetics. Trends Ecol Evol 8: 234-239. 
Nunney L, Elam DR (1994). Estimating the effective population size of conserved populations. Conserv Biol 8: 175-184.

Palstra FP, Ruzzante DE (2008). Genetic estimates of contemporary effective population size: what can they tell us about the importance of genetic stochasticity for wild population persistence? Mol Ecol 17: 3428-3447.

Slatkin M, Barton NH (1989). A comparison of three indirect methods for estimating average levels of gene flow. Evolution 43: 1349-1368.

Vucetich JA, Waite TA, Nunney L (1997). Fluctuating population size and the ratio of effective to census population size. Evolution 51: 2017-2021.

Wang J (1996). Inbreeding and variance effective sizes for nonrandom mating populations. Evolution 50: 1786-1794.

Waples RS (1989). A generalized approach for estimating effective population size from temporal changes in allele frequency. Genetics 121: 379-391.
Waples RS (2005). Genetic estimates of contemporary effective population size: to what time periods do the estimates apply? Mol Ecol 14: 3335-3352.

Whitlock MC, Barton NH (1997). The effective size of a subdivided population. Genetics 146: 427-441.

Wright S (1931). Evolution in Mendelian populations. Genetics 16: 97-159.

Wright S (1938). Size of population and breeding structure in relation to evolution. Science 87: $430-431$.

Wright S (1943). Isolation by distance. Genetics 28: 114-138.

Wright S (1946). Isolation by distance under diverse systems of mating. Genetics 31: 39-59.

Wright S (1951). The genetical structure of populations. Ann Eugenics 15: 323-354.

Yonezawa K (1997). Effective population size of plant species propagating with a mixed sexual and asexual reproduction system. Genet Res 70: 251-258. 